\title{
APPROXIMATION OF DISCRETE AND PENALIZED LEAST SQUARES SPLINES OVER SPHERICAL TRIANGULATIONS
}

\section{HUAXUE ZHOU}

Department of Mathematics

Jinan University

Guangzhou

Guangdong 510632

P. R. China

e-mail: huaxuestudent@163.com

\begin{abstract}
The paper studies spherical polynomial approximation over spherical triangulation. We exhibit the error of the approximation of a sufficiently smooth function using the discrete and penalized least squares splines on the unit sphere and show the effect of grid size on the error of discrete least squares splines of the underlying triangulation. In addition, we also demonstrate that penalty parameters have an effect on the error of the penalized least squares spline from numerical experiments.
\end{abstract}

\section{Introduction}

With the development of digital information technology, the number and dimensions of the data have been changed remarkably. The research of spherical data approximation not only has important theoretical 2010 Mathematics Subject Classification: 97R20.

Keywords and phrases: triangulation, spherical splines, least square splines, penalty parameters.

Received September 18, 2017; Revised November 28, 2017

(ㄷ) 2017 Scientific Advances Publishers 
significance, but also has considerable practical application background. Its application is mainly reflected in the following five aspects: geophysical and seismological research; physical geodesy in the measurement of gravity field; meteorology, oceanography and other related environmental resources data approximation; earth temperature, ozone or magnetic field and other aspects of the study; medical aspects of an EEG analysis.

It has been proved that the spherical spline method is very effective for studying the approximate problem of scattered data. Least square method is a selection of big data sets, including random noise. Therefore, it is necessary to study the approximation of the discrete and penalized least squares spherical triangulation splines in a stable local basis space. The existence and uniqueness of discrete and penalized least squares spherical splines in the $S_{d}^{r}(\Delta)$ are introduced in detail [1].

In this paper, we focus on the unit sphere $\mathbb{S}^{2}:=\left(\mathrm{x}=(x, y, z)^{T} \in \mathbb{R}^{3}\right.$ $\left.\mid x^{2}+y^{2}+z^{2}=1\right\}$. We investigate the approximation properties of discrete and penalized least squares spherical over triangulations. In addition, we show the error of a sufficiently smooth function the discrete and penalized least squares spherical triangulation splines approximation and the effect of the choice of penalty parameters on the error of penalized least squares spherical splines approximation.

In Section 2, we give some preliminary knowledges, including spherical triangulation, spherical Bernstein basis polynomials, spherical splines, and least squares splines. In Section 3, we discuss the existence and uniqueness of discrete least squares splines. In Section 4, we study the properties of the penalized least squares spline and its approximation error. In Section 5, we design some numerical experiments on the error of the smooth function the discrete and penalized least squares splines approximation, and analyze the influence of the choice of the penalty parameters on the error. 


\section{Preliminaries}

Schumaker in $[2,3,4]$ introduced the concept and properties about the approximation of splines on spherical triangulations. Suppose $v_{1}, v_{2}$, $v_{3}$ are three points on the unit sphere which lie strictly in one hemisphere. In [7], the associated spherical triangle $\tau_{i}:=\left\langle v_{1}, v_{2}, v_{3}\right\rangle$ to be the set of points on $\mathbb{S}^{2}$ that lie in the region bounded by the three circular arcs $\left\langle v_{1}, v_{2}\right\rangle,\left\langle v_{2}, v_{3}\right\rangle,\left\langle v_{3}, v_{1}\right\rangle$. The points $v_{1}, v_{2}, v_{3}$ are the vertices of $\tau$, and refer to the circular arcs $\left\langle v_{1}, v_{2}\right\rangle,\left\langle v_{2}, v_{3}\right\rangle,\left\langle v_{3}, v_{1}\right\rangle$ as the edges of $\tau$. The $\tau$ is nondegenerate provided that $\tau$ has nonzero area. $\tau_{i}$ is the spherical triangle on the $\mathbb{S}^{2}$. A set $\Delta:=\left\{\tau_{i}\right\}_{1}^{\mathbb{N}}$ is called a spherical triangulation. $\Delta$ is the sphere $\mathbb{S}^{2}$ or a triangulation on the domain of $\mathbb{S}^{2}$. Arbitrary two spherical triangles in $\Delta$ are either disjoint or exactly intersecting a common edge.

Let $\tau_{i}:=\left\langle v_{1}, v_{2}, v_{3}\right\rangle$ is a nondegenerate spherical triangle. Given $V \in \mathbb{S}^{2}$. Let $b_{i}:=b_{i}(v)$ be such that

$$
v=b_{1}(v) v_{1}+b_{2}(v) v_{2}+b_{3}(v) v_{3}
$$

Then $b_{1}, b_{2}, b_{3}$ are the spherical barycentric coordinates of $v$ relative to $\tau$. It means that the spherical barycentric coordinates of a point relative to $\tau$ are unique.

Given an arbitrary integer $d$, for all $v$ in $\tau$ and every real $r$, there are a formula

$$
f(r v)=r^{d} f(v)
$$

Then the three variables function $f(v)$ is homogeneous of the degree $d$.

Given an integer $d$ and given a spherical triangle $\tau$, the functions

$$
B_{i j k}^{d}(v):=\frac{d !}{i ! j ! k !} b_{1}(v)^{i} b_{2}(v)^{j} b_{3}(v)^{k}, \quad i+j+k=d
$$


are called spherical Bernstein basis polynomials of degree $d$.

Let $\mathcal{B}_{d}:=\operatorname{span}\left\{B_{i j k}^{d}\right\}_{i+j+k=d}$. It asserts that the $\left\{B_{i j k}^{d}\right\}_{i+j+k=d}$ are linearly independent in [6]. Thus, every $p \in \mathcal{B}_{d}$ has a unique representation form

$$
p(v):=\sum_{i+j+k=d} c_{i j k} B_{i j k}^{d}(v),
$$

where $c_{i j k}$ is its spherical $B$-coefficient. $\mathcal{H}_{d}(\tau)$ represents the space of all polynomials. The formula (2.1) is the spherical homogeneous Bernstein basis polynomials of degree $d$.

So as to study the approximation of the least squares spherical splines, we introduce the Sobolev space half-norm.

$$
|f|_{k, p, \Omega}=\sum_{|\alpha|=k}\left\|D^{\alpha} f\right\|_{p, \Omega}
$$

is called the half-norm of $f$ in the Sobolev space $W^{k, p}(\Omega), k \geq 0$, $1 \leq p \leq \infty, \Omega \subseteq \mathbb{S}^{2} . \alpha=\left(\alpha_{1}, \alpha_{2}, \alpha_{3}\right)$ is a three indicators vector, where $|\alpha|=\alpha_{1}+\alpha_{2}+\alpha_{3}, D^{\alpha}$ expresses partial derivative, for example, $D^{(1,1,0)}=D_{x} D_{y}$. The definition and properties of the Sobolev space on the $\mathbb{S}^{2}$ are shown in $[6,8,16]$.

Let us now introduce the spherical spline space. Suppose a points set $\mathcal{V} \in \mathbb{S}^{2}, \Delta$ is a spherical triangulation relative to $\mathcal{V}$. Given integers $d$ and $r$. Let

$$
S_{d}^{r}(\Delta):=\left\{s \in C^{r}\left(\mathbb{S}^{2}\right),\left.s\right|_{\tau} \in H_{d}, \tau \in \Delta\right\}
$$

is the spherical spline space of degree $d$ and smoothness $r$, where

$$
\left.s\right|_{\tau}:=\sum_{i+j+k=d} c_{i j k}^{\tau} B_{i j k}^{d, \tau}(v) .
$$


For some $\beta \geq 0$, a triangulation $\tau$ is called $\beta$-quasi-uniform when

$$
\frac{|\Delta|}{\rho_{\Delta}} \leq \beta
$$

Here $\rho_{\Delta}:=\min \left\{\rho_{\tau}, \tau \in \Delta\right\}$ and $\rho_{\tau}$ is the diameter of the largest spherical cap contained in a spherical triangle $\tau$. The mesh size of the triangulation $\Delta$ is defined by $|\Delta|:=\max \{|\tau|, \tau \in \Delta\}$.

\section{Discrete Least Squares Splines}

Let $\mathcal{V}=\left\{v_{i}, i=1, \ldots, n\right\}$ be a given data sites on $\mathbb{S}^{2}$ and $\Delta$ be a triangulation with $\mathbb{S}^{2}$. Fixed two integers $d>r \geq 0$, the spherical spline space of degree $d$ and smoothness $r$ of $\Delta$ is $S_{d}^{r}(\Delta)$. The set $f(v), v \in \mathcal{V}$ is the data values of a given function $f$. The least squares functional is defined by

$$
\mathcal{L}(s):=\sum_{v \in V}|s(v)-f(v)|^{2}
$$

The discrete least squares spline $s_{0, f} \in S_{d}^{r}(\Delta)$ is the minimum value of the Equation (3.3), i.e.,

$$
\mathcal{L}\left(s_{0, f}\right)=\min \left\{\mathcal{L}(s): s \in S_{d}^{r}(\Delta)\right\}
$$

Suppose $\mathcal{V}=\left\{v_{i}, i=1, \ldots, n\right\}$ be the data sites on $S_{d}^{r}(\Delta)$, if for any $\tau \in \Delta$, the matrix $\left\{B_{i j k}^{d, \tau}\left(v_{i}\right)\right\}_{i+j+k=d}, v_{i} \in \tau$ is full rank. Then the set $\mathcal{V}$ is evenly distributed on the triangulation relative to the degree $d$.

The existence and uniqueness theorem of discrete least squares spline are given in the following:

Theorem 3.1 ([9]). Let the set $\mathcal{V}$ be evenly distributed on the triangulation relative to the degree $d$. There exists a unique spline $s_{0, f}$ of degree $d$ and smoothness $r$ such that the value of 3 is the minimal. 
Theorem 3.2 ([9]). Suppose the set $\mathcal{V}$ be evenly distributed over $S_{d}^{r}(\Delta)$. The triangulation $\Delta$ is quasi-uniform. Here $d \geq 3 r+2$ and $|\Delta| \leq 1$. Then there exist a constant $C$ depending on the the spline space and distribution of the data locations such that for any function $f$ in the Sobolev space $W^{k, p}\left(\mathbb{S}^{2}\right)$

$$
\left\|f-s_{0, f}\right\|_{\infty, \mathbb{S}^{2}} \leq C|\Delta|^{m+1}|f|_{m+1, \infty, \mathbb{S}^{2}}
$$

where $0<m<d$ and $(d-m) \bmod 2=0, m \in \mathbb{Z}$.

Note that $|\Delta|:=\max \{|\tau|, \tau \in \Delta\}$ denotes the mesh size of the triangualtion $\Delta$. It is known that the approximation error of the discrete least square spine depended on the mesh size of the triangulation. In addition, the error of the discrete least squares spline is related to the constant $C$, which relies on the nature of the spline space and the distribution of the data sites.

\section{Approximation of Penalized Least Squares Splines}

Suppose $\mathcal{V}=\left\{v_{i}, i=1, \ldots, n\right\}$ be a given data sites on $\mathbb{S}^{2}$ and $\Delta$ be a triangulation with $\mathbb{S}^{2}$. Fix two integers $d>r \geq 0$, the spherical spline space of degree $d$ and smoothness $r$ of $\Delta$ is $S_{d}^{r}(\Delta)$. The set $f(v), v \in \mathcal{V}$ is the data values of a given function $f$. Fix a parameter $\lambda>0$, we find a spline solution $s_{\lambda, f} \in S_{d}^{r}(\Delta)$ satisfying

$$
P_{\lambda}\left(s_{\lambda, f}\right)=\min \left\{P_{\lambda}(s): s \in S_{d}^{r}(\Delta)\right\}
$$

Here

$$
P_{\lambda}(s):=\mathcal{L}(s-f)+\lambda \varepsilon(s)
$$

is the penalized least square spline function. $\mathcal{L}$ is the discrete least square spline function, and $\varepsilon$ is energy function which is defined by 


$$
\varepsilon(s):=\int_{\mathbb{S}^{2}} \sum_{|\alpha|=2}\left|D^{\alpha} s\right|^{2},
$$

where $D^{\alpha} s,|\alpha|=2$ denotes the second order partial derivative of the spline $s$. More precisely, $s$ is homogeneous and differentiable.

The situation is a discrete least squares fit if $\lambda=0, s_{0, f}$. Therefore, we additionally require that the data sites are evenly distributed over $S_{d}^{r}(\Delta)$.

Theorem 4.3 ([9]). Given a parameter $\lambda>0$. Let the set of the vertices $\mathcal{W}$ of $\Delta$ is a subset of the data sites $\mathcal{V}$. The size of every triangle is satisfied $|\tau| \leq 1$. Then there exist a unique spline $s_{\lambda, f} \in S_{d}^{r}(\Delta)$ minimizing (4.5).

Theorem 4.4 ([9]). Suppose $\Delta$ is a $\beta$-quasi-uniform triangulation over $S_{d}^{r}(\Delta)$ and the mesh size of $\Delta$ is bounded above by $1 . \mathbb{N}$ denotes the number of the triangle in $\Delta$. Let the triangulation satisfies two conditions:

(1) the set $\mathcal{V}$ be evenly distributed over $S_{d}^{r}(\Delta)$ with $d \geq 3 r+2$;

(2) the set of the vertices $\mathcal{W}$ of $\Delta$ is a subset of the data sites $\mathcal{V}$,

$s_{\lambda, f}$ is a spline $s_{\lambda, f} \in S_{d}^{r}(\Delta)$ minimizing 5. Then for every $f \in W^{k, p}\left(\mathbb{S}^{2}\right)$, there exist the constant $C$ relies on the nature of the spline space and the distribution of the data sites such that

$\left\|f-s_{\lambda, f}\right\|_{\infty, \mathbb{S}^{2}} \leq C|\Delta|^{m+1}|f|_{m+1, \infty, \mathbb{S}^{2}}+\lambda \mathbb{N}\left(C_{1}|\Delta|^{M-1}|f|_{m+1, \infty, \mathbb{S}^{2}}+C_{2}|f|_{2, \infty, \mathbb{S}^{2}}\right)$,

where $W^{k, p}\left(\mathbb{S}^{2}\right)$ is the Sobolev space. The integer $m$ is claimed to fulfill $1<m<d$ and $(d-m) \bmod 2=0$. 
According to Theorem 4.4, the error of a smooth function the penalized least squares splines approximation depends on the constant coefficients. And the constant coefficient depends on the nature of the spline space and the distribution of the data points. The error is also related to the selection of penalty parameters.

\section{Numerical Experiments}

In this section, we give some examples of a smooth function the discrete and penalized least squares splines approximation. We show that the error of the smooth function the discrete least squares splines approximation is related to the mesh size of triangulation in Example 1. We expain that the error of the smooth function the penalized least squares splines approximation is relied to the parameter $\lambda$ in Example 2.

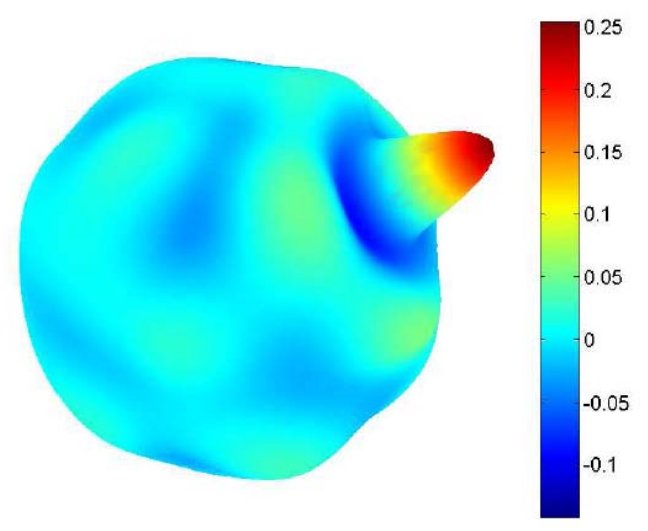

Figure 1. Error in $S_{5}^{1}\left(\Delta_{0}\right), \max =2.535 \mathrm{e}-1$. 


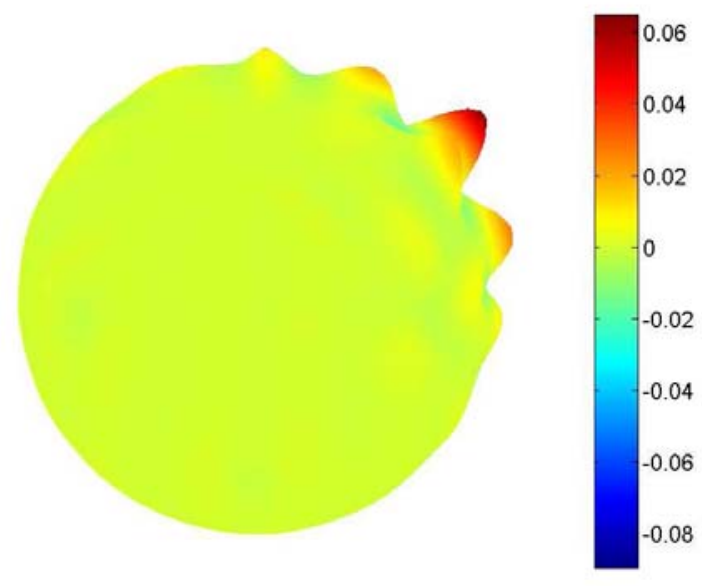

Figure 2. Error in $S_{5}^{1}\left(\Delta_{1}\right), \max =8.98 \mathrm{e}-2$.

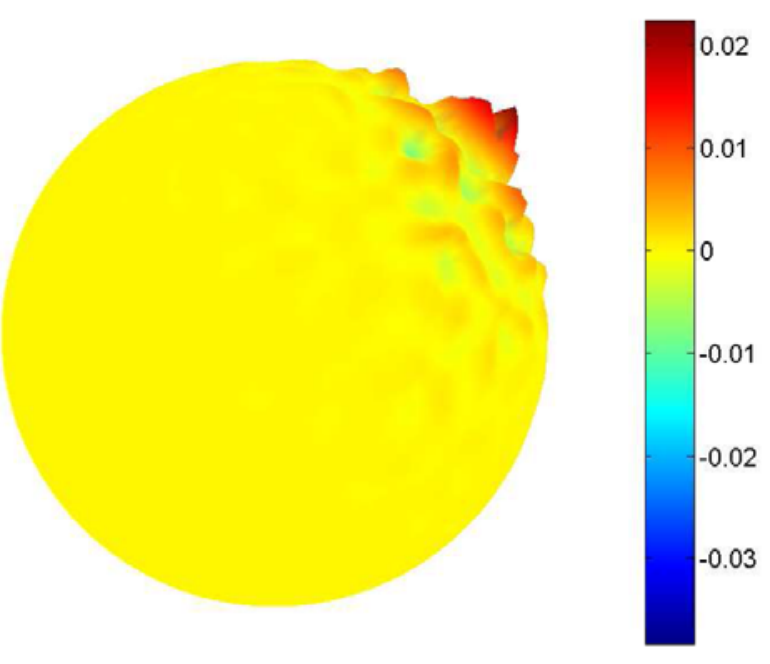

Figure 3. Error in $S_{5}^{1}\left(\Delta_{2}\right), \max =3.83 \mathrm{e}-2$. 


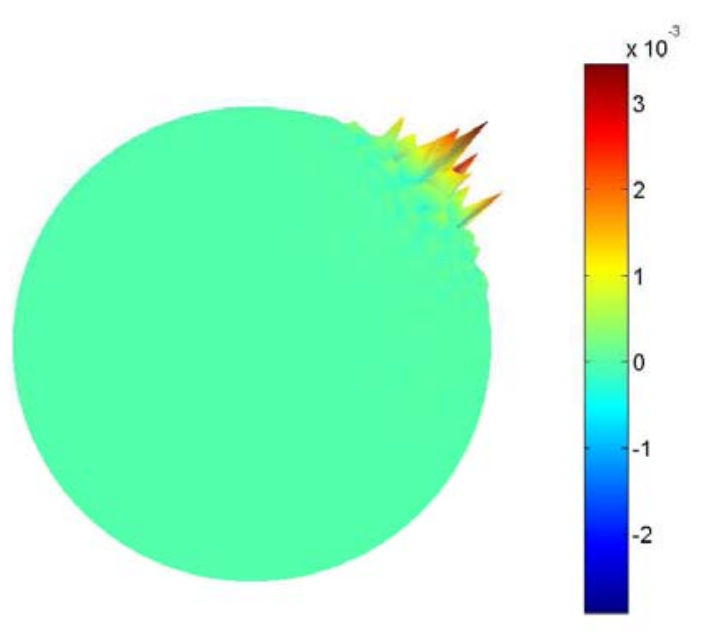

Figure 4. Error in $S_{5}^{1}\left(\Delta_{3}\right), \max =3.4 \mathrm{e}-3$.

Example 1. Let $\Delta_{0}$ be a triangulation of the unit sphere $\mathbb{S}^{2}$ with 8 triangles and 6 vertices $(1,0,0)^{T},(0,1,0)^{T},(0,0,1)^{T},(-1,0,0)^{T}$, $(0,-1,0)^{T},(0,0,-1)^{T}$. It defines $\Delta_{0}$ uniformly to get $\Delta_{1}$ by partitioning each triangle into four using midpoints of the edges. Similarly, $\Delta_{k+1}$ denotes the uniform refinement of $\Delta_{k}$ for $k=1,2$, 3. We use a set of 4,3200 data sites almost uniformly scattered on the sphere and use the smooth Franke [14] function as a test function

$$
\begin{aligned}
f(\mathrm{x}) & =0.75 \exp \left(-\frac{(9 x-2)^{2}}{4}-\frac{(9 y-2)^{2}}{4}-\frac{(9 z-2)^{2}}{4}\right) \\
& +0.75 \exp \left(-\frac{(9 x+1)^{2}}{49}-\frac{(9 y+1)}{10}-\frac{(9 z+1)}{10}\right) \\
& +0.5 \exp \left(-\frac{(9 x-7)^{2}}{4}-\frac{(9 y-3)^{2}}{4}-\frac{(9 z-5)^{2}}{10}\right) \\
& +0.2 \exp \left(-(9 x-4)^{2}-(9 y-7)^{2}-(9 z-5)^{2}\right), \quad \mathrm{x}=(x, y, z)^{T} \in \mathbb{S}^{2} .
\end{aligned}
$$


There are a discrete least squares spline $s_{0, f} \in S_{d}^{r}(\Delta)$ by using the iterative algorithm in [9]. Here we select $d=5, r=1$ and calculate the maximum values of the error $\left|f-s_{0, f}\right|$. Figures 1-4, respectively, represent the error of the discrete least squares spline $s_{0, f}$ in $S_{5}^{1}\left(\Delta_{0}\right), S_{5}^{1}\left(\Delta_{1}\right), S_{5}^{1}\left(\Delta_{2}\right)$, and $S_{5}^{1}\left(\Delta_{3}\right)$. From the analysis of the results of the image, we can see that the error of the smooth function the discrete least squares splines approximation decreases with the increase of the number of the uniform refinement of the triangulation.

Table 1. The maximum values $e_{5, k}, k=0,1,2,3$ with different parameter $\lambda$

\begin{tabular}{ccccc}
\hline$\lambda \backslash e_{5, k}$ & $e_{5,0}$ & $e_{5,1}$ & $e_{5,2}$ & $e_{5,3}$ \\
\hline$\lambda=e-1$ & $2.390 \mathrm{e}-1$ & $9.980 \mathrm{e}-2$ & $3.830 \mathrm{e}-2$ & $3.400 \mathrm{e}-3$ \\
$\lambda=e-2$ & $2.585 \mathrm{e}-1$ & $9.350 \mathrm{e}-2$ & $3.950 \mathrm{e}-2$ & $3.500 \mathrm{e}-3$ \\
$\lambda=e-3$ & $2.535 \mathrm{e}-1$ & $8.980 \mathrm{e}-2$ & $3.830 \mathrm{e}-2$ & $3.400 \mathrm{e}-3$ \\
$\lambda=e-4$ & $2.585 \mathrm{e}-1$ & $9.350 \mathrm{e}-2$ & $3.950 \mathrm{e}-2$ & $3.500 \mathrm{e}-3$ \\
$\lambda=e-5$ & $2.535 \mathrm{e}-1$ & $8.980 \mathrm{e}-2$ & $3.830 \mathrm{e}-2$ & $3.400 \mathrm{e}-3$ \\
$\lambda=e-6$ & $2.585 \mathrm{e}-1$ & $9.350 \mathrm{e}-2$ & $3.950 \mathrm{e}-2$ & $3.500 \mathrm{e}-3$ \\
\hline
\end{tabular}

Example 2. In this example, we consider the approximation of the PLS in the spline space $S_{5}^{1}\left(\Delta_{k}\right), k=0,1,2,3$. We choose the testing function $h=x^{5}+2 x$ on 4,3200 scattered data in example 1 . We compute the PLS splines $s_{\lambda, h}$ with the different parameter $\lambda$. The maximum values $e_{5, k}, k=0,1,2,3$ with the different parameter $\lambda>0$ are listed in Table 1 . The results suggest that the errors on each $\Delta_{k}$ are almost the same for all the values of $\lambda$. 


\section{Final Remark}

This paper is aimed at explaining the errors $e_{d, k}$ for each of the computed splines over triangulation $\Delta_{k}$. In addition, we find that the errors on each $\Delta_{k}$ are almost the same for all the values of $\lambda$. In the next step, we can study the relation of the errors on different $\Delta_{k}$ for different value of $\lambda$. In the future, we will compare the method in this paper with the regularization least square approximation over $\mathbb{S}^{2}[19]$.

\section{Acknowledgement}

I would like to express my gratitude to all those who helped me during the writing of the paper. For his encouragement and help, comments and advice, I am indebted to Professor Congpei An. Thanks are also due to Professor Victoria Baramidze and Professor Mingjun Lai for sharing their codes.

\section{References}

[1] V. Baramidze, M. J. Lai and C. K. Shum, Spherical splines for data interpolation and fitting, SIAM J. Sci. Comput. 28 (2006), 241-259.

[2] P. Alfeld, M. Neamtu and L. L. Schumaker, Fitting scattered data on sphere-like surfaces using spherical splines, J. Comp. Appl. Math 73 (1996), 5-43.

[3] P. Alfeld, M. Neamtu and L. L. Schumaker, Bernstein-Bezier polynomials on spheres and spheres and sphere-like surface, Comput. Aided Geom. Design 13 (1996), 333-349.

[4] P. Alfeld, M. Neamtu and L. L. Schumaker, Dimension and local bases of homogeneous spline spaces, SIAM J. Math. Anal. 27 (1996), 1482-1501.

[5] V. Baramidze and M. J. Lai, Error bounds for minimal energy interpolatory spherical splines, In: C. K. Chui, M. Neamtu, L. L. Schumaker (eds.), Approximation Theory XI, Nashboro Press, Brentwood, (2005), 25-50.

[6] M. J. Lai and L. L. Schumaker, Splines Functions over Triangulations, Cambridge University Press, Cambridge, 2007.

[7] M. von Golitschek and L. L. Schumaker, Bounds on projections onto bivariate polynomial spline with stable local bases, Constr. Approx. 18 (2002), 241-254. 
[8] M. Neamtu and L. L. Schumaker, On the approximation order of splines on spherical triangulations, Adv. Comput. Math. 21 (2004), 3-20.

[9] V. Baramidze and M. J. Lai, Convergence of discrete and penalized least squares spherical splines, In: Journal of Approximation Theory. 163 (2011), 1091-1106.

[10] V. Baramidze, M. J. Lai, C. K. Shum and P. Wenston, Triangulated spherical splines for geopotential reconstruction, J. Geod. 83(8) (2008), 695-708.

[11] M. von Golitschek and L. L. Schumaker, Penalized least squares fitting, In: Algorithms for Approximation II, Chapman and Hall, London, 1990.

[12] M. von Golitschek and L. Schumaker, Penalized least squares fitting, Serdica 18 (2002), 1001-1020.

[13] H. Wendland and C. Rieger, Approximate interpolation with applications to selecting smoothing parameters, Numer. Math. 101 (4) (2005), 729-748.

[14] R. J. Renka, Multivariate interpolation of largesets of scattered data, J. ACM Trans. Math. Soft-Ware (TOMS) 14 (1988), 139-148.

[15] V. Baramidze, Spherical splines for scattered data fitting Ph.D. Disseratation Department of Mathematics the University of Georiga, Athens, GA, 2005.

[16] R. Adams, Sobolev spaces, Academic Press, New York, 1975.

[17] G. Fasshauer and L. L. Schumaker, Scattered data fitting on the sphere in Mathematical Methods for Curves and Surfaces II, M. Daehlen, T. Lyche, L. Schumaker, Vanderbilt University Press, (1998), 117-166.

[18] G. Wahba, Splines for Observation ata, SIAM Publication, Philadelphia, 1990.

[19] C. P. An, X. J. Chen, I. H. Sloan and R. S. Womersley, Regularized least squares approximation on the sphere using spherical designs, J. SIAM Journal on Numerical Analysis 50 (2012), 1513-1534. 